\title{
Numerical study of a Solar Absorption Refrigeration Machine
}

\author{
J.Dardouch ${ }^{1, *}$, M. Charia $^{1,2}$, A. Bernatchou ${ }^{1,2}$ \\ ${ }^{1}$ Laboratory of Solar Energy and Environment, Department of Physics, faculty of Sciences, Mohammed V University, \\ BP.1014.Rabat, Morocco \\ ${ }^{2}$ Department of Physics, Team thermodynamic energy, Faculty of sciences, Mohammed V University, Raba B.P. 1014, Rabat, \\ Morocco
}

\begin{abstract}
In this paper, we present a numerical study of a single-stage absorption refrigeration machine, operating with a couple of water-ammonia fluids, equipped with a distillation column and associated with a solar heating system using solar collectors.

The study has showed the benefit of using the distillation column which is manifested by:

- The decrease of the operating temperature,

- The improvement of the coefficient of performance,

- Surface reduction of the solar collectors,

- The improvement of the solar coefficient of performance.

The solar study shows that the absorption refrigeration machine equipped with a distillation column is better suited to solar energy with significantly better performance compared to the simple absorption refrigeration machine.
\end{abstract}

\section{Introduction}

Environmental pollution and the energy crisis have led in recent years to many studies on the rational use of energy[1].

Especially in the field of industrial refrigeration which exhausts a large part of energy in air conditioning [1.2].

Absorption refrigeration machines use a mixture of fluids (Absorbent fluid - Refrigerant fluid) for their operation. In order to select the best mixture of active fluids, several mixtures of CFCs (chloro-fluorocarbons) with organic absorbers were studied [3-6]. But because CFCs have a destructive effect on atmospheric ozone, the mixture of water-ammonia fluids remains the best, especially if one is trying to produce cold at temperatures below zero degrees Celsius as well as because of its low price [7-10].

Since the Montreal Protocol (1987), CFC and HCFC refrigerants have been progressively replaced (most often by HFCs) and the production of these fluids has been discontinued (CFC) or severely restricted (HCFCs). But the Kyoto Protocol (1997) points to these first HFC-type refrigerants (which are classified as greenhouse gases) and therefore aims to reduce the fluid load in refrigeration plants.

In addition, Morocco has a significant solar potential. Annual sunshine is always above $20000 \mathrm{kj} \mathrm{per} \mathrm{m}^{2}$ of collector area.it is therefore important to exploit this deposit, free and non-polluting, in the field of cold production, especially in remote rural areas. The market for solar absorption refrigeration machine installations is growing, and the quantity of the energy used for cooling buildings has greatly increased in recent years [11-20].

The present work deals with the study of a solar absorption (water-ammonia) refrigeration machine equipped with a distillation column. We have realized a program of simulation of the machine, based on the laws of conservation of the mass and the energy applied at the level of each element of the machine and actual operating conditions of the machine, and the thermodynamic properties of the ammonia mixture. And real solar data from the Rabat site(Morocco).

The results of the numerical simulation of the absorption machine with and without a distillation column show a gain on the coefficient of performance of $30 \%$. The optimum operating temperature of the absorption machine at the generator has been reduced by twenty-five degrees, which influences the choice of appropriate collectors for the operation of the machine and makes it possible subsequently to use simple flat plate collectors less expensive and available in the market, while the absorption machine without distillation column requires for its operation evacuated tube collectorsexcessively expensive and less available in the markets of developing countries.

\section{Principle of operation of the solar powered absorption machine}

The main components of the solar absorption refrigeration machine are solar thermal collector, an evaporator, an absorber, generator, distillation column,

Corresponding author: $\underline{\text { dardouch81@gmail.com }}$ 
a condenser, an expansion valve, a heat exchanger and a pump. A simplediagram of the solar absorption refrigeration machine is shown in Figure 1. Two kinds of working medium are used at the same time in refrigeration and absorption processes.

In this machine the solar thermal collector collects heat by absorbing sunlight. The heat collected in the solar collector is transferred to the generator and it is used for heating an ammonia-water solution and send the vapour to the condenser. Remaining weak solution flows to the absorber through heat exchanger where the heat is transferred to the strong solution. Liquid refrigerant from the condenser goes through an expansion valve while the pressure is decreased to an effect is achieved by the vaporization of the refrigerant at a low temperature. Refrigerant vapor from the evaporator continues to an absorber and dissolves in a weak refrigerant solution, and it becomes a stronger refrigerant solution, which is called "rich solution". A pump is the only moving part in this system. The "rich solution" is pumped to a generator. At the generator, the rich solution is heated up; the refrigerant is separated from the solution. The refrigerant is vaporized and goes to the condenser while the weak solution is passed through a heat exchanger and returned to the absorber to absorb the refrigerant vapour. The refrigeration process and the regeneration process operate at the same time as the continuous process, producing a continuous cooling effect. A simple flat plate collector can maintain the operating condition at the generation temperature[2122].

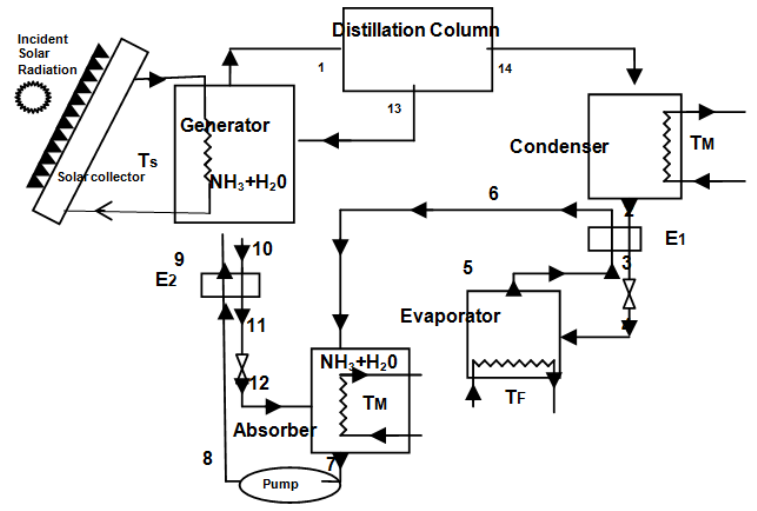

Fig. 1.Schematic representation of the solarabsorption refrigeration machine with distillation column.

\section{Mass and energy balances of the different elements}

\subsection{Distillation column}

The conservation of the total mass is given by:

$$
q m_{14}+q m_{13}-q m_{1}=0
$$

The conservation of mass by element is expressed as follows:

$$
q m_{14} \cdot Y_{14}+q m_{13} \cdot X_{13}-q m_{1} \cdot Y_{1}=0
$$

The enthalpy balance of the distillation column is given by:

$$
q m_{14} \cdot h_{14}+q m_{13} \cdot h_{13}-q m_{1} \cdot h_{1}=Q_{D C}
$$

With $Q_{D C}$ : The amount of heat supplied to the distillation column

This system of equations gives:

$$
\left\{\begin{array}{l}
q m_{14}+q m_{13}-q m_{1}=0 \\
q m_{14} \cdot Y_{14}+q m_{13} \cdot X_{13}-q m_{1} \cdot Y_{1}=0
\end{array}\right.
$$

The resolution of this system of equations gives:

$$
\begin{aligned}
q m_{13} & =q m_{1} \cdot\left(\frac{Y_{14}-Y_{1}}{Y_{14}-X_{13}}\right) \\
q m_{14} & =q m_{1} \cdot\left(\frac{Y_{1}-X_{13}}{Y_{14}-X_{13}}\right)
\end{aligned}
$$

From this system of equations we can be deduce the expression of the quantity of heat supplied to the distillation column

$$
\frac{Q_{D C}}{q m_{1}}=\left(h_{14}-h_{1}\right)+\left(h_{13}-h_{14}\right) \frac{\left(Y_{14}-Y_{1}\right)}{\left(Y_{14}-X_{13}\right)}
$$

\subsection{We can summarize the heat flow of the machine as follows}

Using mass balances, energetic and enthalpic balances

\begin{tabular}{|c|c|}
\hline \multicolumn{2}{|c|}{ Generator } \\
\hline \multicolumn{2}{|c|}{$Q_{G}=q m_{1} \cdot h_{1}+q m_{10} \cdot h_{10}-q m_{13} \cdot h_{13}-q m_{9} \cdot h_{9}$} \\
\hline \multicolumn{2}{|c|}{ Absorber } \\
\hline \multicolumn{2}{|c|}{$Q_{A}=q m_{7} \cdot h_{7}-q m_{12} \cdot h_{12}-q m_{14} \cdot h_{14}$} \\
\hline Condenser & Evaporator \\
\hline$Q_{c}=q m_{14}\left(h_{2}-h_{14}\right)$ & $Q_{F}=q m_{14}\left(h_{5}-h_{4}\right)$ \\
\hline Exchanger $_{E_{1}}$ & Exchanger $_{E_{2}}$ \\
\hline$h_{6}-h_{5}=h_{2}-h_{3}$ & $q m_{9}\left(h_{9}-h_{8}\right)=q m_{10}\left(h_{10}-h_{11}\right)$ \\
\hline Regulator $_{D_{\mathrm{i}}}$ & Regulator $_{D_{2}}$ \\
\hline$h_{4}=h_{3}$ & $h_{12}=h_{11}$ \\
\hline Pump & Distillation column \\
\hline$P_{p}=V_{r} \cdot \frac{\left(P_{\mathrm{s}}-P_{7}\right)}{\eta_{p}}$ & $Q_{D C}=q m_{14} \cdot h_{14}+q m_{13} \cdot h_{13}-q m_{1} \cdot h_{1}$ \\
\hline
\end{tabular}
on various machine components and operational hypotheses, we find the expression of the heat flux at the levels of the different organs of the machine[22]:

Table 1.The heat flows of the various units of the single stage absorption refrigeration machine with distillation column.

\footnotetext{
Corresponding author: dardouch81@,gmail.com
} 
Thus, by determining the thermodynamic properties (temperature, pressure, composition, enthalpy, entropy, specific volume, vapor and liquid titre) of the water-ammonia fluid pair, the method is based on equilibrium quantities (temperature, pressure, composition), determined using the Peng-Robinson state equation, and the specific volume, entropy and enthalpy at each point of the machine cycle is calculated from the analytical expression of the Gibbs free energy given by Ziegler, The high and the low pressure are calculated by the formula of Antoine, we can determine the coefficient of performance (COP) of the refrigeration machine with absorption[23-29].

$$
C O P=\frac{Q_{F}}{Q_{G}}=\frac{q m_{14}\left(h_{5}-h_{4}\right)}{q m_{1} \cdot h_{1}+q m_{10} \cdot h_{10}-q m_{13} \cdot h_{13}-q m_{9} \cdot h_{9}}
$$

The coefficient of performance of the absorption machine becomes:

$$
\begin{aligned}
C O P= & \frac{\left(h_{5}-h_{4}\right) \cdot\left(\frac{Y_{1}-X_{13}}{Y_{14}-X_{13}}\right)}{\left(h_{1}-h_{9}\right)+\left(\frac{Y_{14}-Y_{1}}{Y_{14}-X_{13}}\right) \cdot\left(\frac{Y_{1}-X_{13}}{X_{10}-X_{9}}\right) \cdot h 9+\left[\frac{X_{9}-Y_{1}}{X_{10}-X_{9}}+\left(\frac{Y_{14}-Y_{1}}{Y_{14}-X_{13}}\right) \cdot\left(\frac{X_{13}-X_{9}}{X_{10}-X_{9}}\right)\right] h_{10+}} \\
& \left(\frac{Y_{14}-Y_{1}}{Y_{14}-X_{13}}\right) \cdot h_{9}
\end{aligned}
$$

The efficiency of a collector depends on its temperature.The higher the temperature of a given collector, the greater its heat loss. In designing solar absorption refrigeration, the collector efficiency $\left(\eta_{s}\right)$, which is defined as the ration of the output $\left({ }_{Q_{G}}\right)$ to the incident solar power $\left(I_{S}\right)$, is very important:

$$
\eta_{s}=\frac{Q_{G}}{Q_{i}}
$$

Taking into account the temperatures reached by solar collectors, we have selected the evacuated tube collectors. The efficiency of which is given by Hottelwhilier:

$$
\eta_{S}=F_{B}\left(\eta-\frac{u\left(T_{S}-T_{M}\right)}{I_{S}}\right)
$$

Where

$$
F_{B}=F_{R}\left(1-F_{R} \beta\right)^{-1}, F_{R}=\frac{1-\exp (-\beta)}{\beta}, \beta=\frac{\mu}{m c_{P}}
$$

With the following characteristics: $\eta=0,7$ is the optical efficiency, $u=2 \mathrm{~W} / \mathrm{m}^{2} \mathrm{~K}$ is the coefficient of thermal losses, $m=50 \mathrm{Kg} / \mathrm{hm}^{2}$ is the flow rate by surface unit, $I_{S}=800 \mathrm{~W} / \mathrm{m}^{2}$ is the global irradiation of an inclined plane, $c_{P}=4186 \mathrm{~J} / \mathrm{Kg} \cdot \mathrm{K}^{\text {is }}$ the specific heat of working fluid (water), $T_{S}$ is the temperature of heat source and $T_{M}$ is the condenser temperature.

The overall $C O P_{S}$ of the solar absorption refrigeration is equal to the product of the efficiency of the solar collector and the absorption refrigeration given equation [30].

$$
C O P_{S}=\eta_{S} \cdot C O P
$$

\section{Test conditions in Morocco}

The measurements used in this work were carried out in the laboratory of solar energy and environment station of the Faculty of Sciences of Rabat Figure 2. The city of Rabat, latitude $34^{\circ} 02^{\prime}$ North and longitude $6^{\circ} 51^{\prime}$ West.

The station has been equipped with complementary instruments for measuring the spectral components. The station provides three types of measurements: the usual solar components (global, direct and diffuse), the solar spectral components and the climatic variables. These measurements are managed by a data acquisition and storage system.

The data acquisition program that we have developed can perform, for each solar component, flow measurements (in $\mathrm{W} / \mathrm{m}^{2}$ ) at a pace of five seconds; it calculates the sum of the measurements made for one hour and finally records a mean hourly irradiation. Thus, each hourly average represents an energy expressed in $\mathrm{Wh} / \mathrm{m}^{2}$ since it corresponds to the product of an average flow in $\mathrm{W} / \mathrm{m}^{2}$ per hour of integration $[31,32]$.

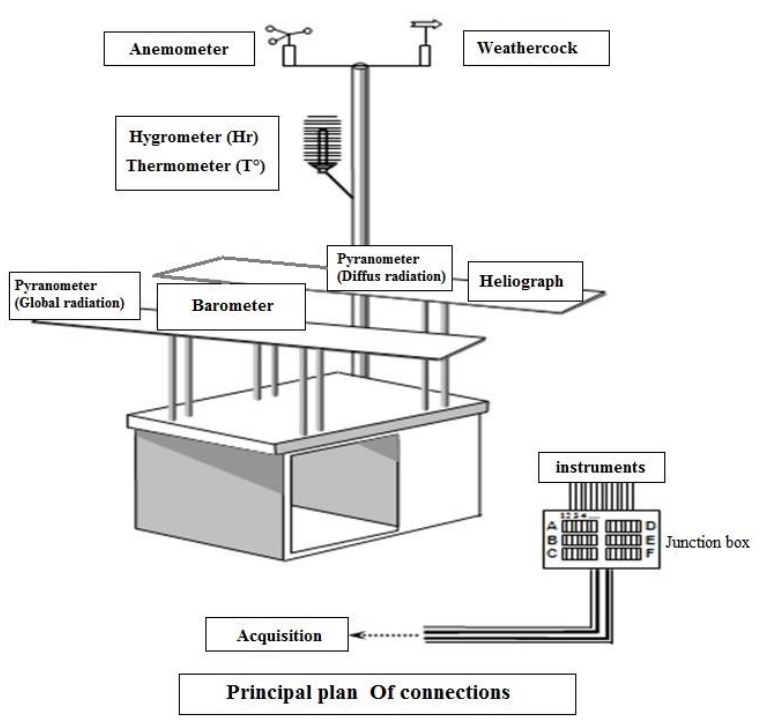

Fig. 2.Diagram of the measuring station located at the Laboratory of Solar Energy and Environment 


\section{Main refrigerants}

Since the invention of absorption refrigeration machines several refrigerants were used, Table 2 shows some examples of used couples, it is noted that the most common is the $\mathrm{H}_{2} \mathrm{O}-\mathrm{LiBr}$ couple and the oldest is the $\mathrm{NH}_{3}-\mathrm{H}_{2} \mathrm{O}$ couple. These couples are the best known.

Table 2.couples used for absorption machines.

\begin{tabular}{|l|l|}
\hline \multicolumn{1}{|c|}{ Refrigerants } & \multicolumn{1}{c|}{ Absorbents } \\
\hline Ammonia & Water \\
\hline Water & Lithium Bromide \\
\hline $\mathrm{R}_{22}$ & Dimethylether Tetraethylene Glycol \\
\hline Methylamine & Water \\
\hline Methanol & Lithium Bromide \\
\hline
\end{tabular}

\section{Thermodynamic study of the properties of the solution $\left(\mathrm{NH}_{3}-\mathrm{H}_{2} \mathrm{O}\right)$}

The determination of the thermodynamic properties of the water-ammonia solution and enthalpy, the temperature, the pressure, the composition, entropy, specific volume, vapor and liquid titer is based on equilibrium quantities (temperature, pressure, composition ) determined using Peng Robinson's state equation.

The specific volume, entropy and enthalpy at each point of the machine cycle are calculated using the analytical expression of the free Gibbs energy given by Ziegler, and the high and low pressure that are calculated by the formula of Antoine[33-36].

\subsection{Analytical expressions of enthalpy,}

\section{entropy and specific volume $\left(\mathrm{NH}_{3}-\mathrm{H}_{2} \mathrm{O}\right)$}

From all the experimental data of the waterammonia pair which it had and from Viriel's equation of state:

$$
P V=R T+B(T) \cdot P(13)
$$

With

$$
B(T)=R \cdot\left(a_{1} \cdot T+a_{2}+\frac{a_{3}}{T}\right)(14)
$$

For the vapor phase, as well as the equation of state:

$$
V(T, P)=C_{1}+C_{2} \cdot P+C_{3} \cdot T+C_{4} \cdot T^{2}(15)
$$

For the liquid phase, Schultz has gaven analytic expressions of Gibbs' free energy as a function of temperature, pressure and title:

$$
G=G(T, P, X)(16)
$$

These equations give a good representation of the experimental data in the temperatures range from 200 to $450 \mathrm{k}$ and at pressures range from 0.01 to $25 \mathrm{bar}$.

Based on the Schultz equations, B. Ziegler and Ch, Trepp, have established other analytic expressions of Gibbs free energy. The area of validity has been extended:

$$
\begin{gathered}
230 \mathrm{~K}<\mathrm{T}<500 \mathrm{~K} \\
0,2 \mathrm{bar}<\mathrm{P}<50 \text { bars }
\end{gathered}
$$

From these expressions of the Gibbs free energy and the specific heats $\mathrm{Cp}$ all the other thermodynamic quantities can be determined by differentiation techniques, notably the enthalpy, the entropy and the volume which are the ones that interest us:

\subsubsection{Liquid state}

$V^{l}=\left(\frac{\partial G^{\prime}(T, P, X)}{\partial p}\right)_{T, X}, H^{l}=-T^{2}\left(\frac{\frac{\partial\left(G^{\prime}(T, P, X)\right.}{T}}{\partial T}\right)_{P, X}, S^{l}=-\left(\frac{\partial G^{\prime}(T, P, Y)}{\partial T}\right)_{P, X}$

\subsubsection{Gaseous state}

$$
V^{V}=\left(\frac{\partial G^{v}(T, P, X)}{\partial p}\right)_{T, Y},
$$

$$
\begin{array}{r}
H^{V}=-T^{2}\left(\frac{\frac{\partial\left(G^{V}(T, P, Y)\right)}{T}}{\partial T}\right)_{P, Y}^{(19)} \\
S^{l}=-\left(\frac{\partial G^{V}(T, P, Y)}{\partial T}\right)_{P, Y}
\end{array}
$$

To make the equations adimensional, they have introduced the following reduced variables:

$T_{r}=\frac{T}{T_{0}}, P_{r}=\frac{P}{P_{0}}, C_{r}=\frac{G}{R \cdot T_{0}},(21)$

$C_{\mathrm{Pr}}=\frac{C_{P}}{R}, \quad H_{r}=\frac{H}{R \cdot T_{0}}, S_{r}=\frac{S}{R}(22)$

The values of the constants $T_{0}, P_{0}$ and $R$ are:

\footnotetext{
*Corresponding author: dardouch81@gmail.com
} 
$T_{0=100 \mathrm{~K},} P_{0=10 \text { bars, }} R=8,3143 \mathrm{Kj} /$ (k.mole.k)

\subsection{Pure state}

\subsubsection{Liquid phase:}

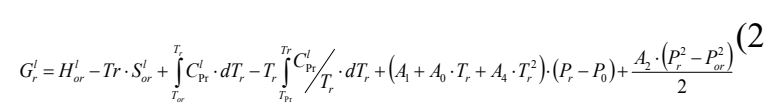

With

$C_{\mathrm{Pr}}^{l}=B_{1}+B_{2} \cdot T_{r}+B_{3} \cdot T_{r}^{2}(24)$

\subsubsection{Gas phase:}

$$
\begin{aligned}
& G_{r}^{V}=H_{o r}^{V}-T_{r} \cdot S_{o r}^{V}+\int_{T_{o r}}^{T_{r}} C_{\mathrm{Pr}}^{V} \cdot d T_{r}-T_{r} \int_{T_{o r}}^{T_{r}} \frac{C_{\mathrm{Pr}}^{V}}{T_{r}} \cdot d T_{r}+\ln \left(\frac{P_{r}}{P_{0 r}}\right) \\
& +{ }^{C} \cdot\left(P_{r}-P_{o r}\right)+C_{2} \cdot\left(P_{r} / T_{r}^{3}-4 \cdot P_{o r} / T_{o r}^{3}+3 P_{o r} \cdot T_{r} / T_{o r}^{4}\right) \\
& +C^{C_{3} \cdot} \cdot P_{r} / T_{r}^{11} \cdot-12 \cdot P_{o r} / T_{o r}^{11+11 P_{o r}} \cdot \frac{T_{r}}{T_{r}^{12}}
\end{aligned}
$$

$$
+{ }^{C 4} \cdot \frac{P_{r}^{3} / T_{r}^{11-12} \cdot P_{o r}^{3} / T_{o r}^{11}+11 P_{o r}^{3} \cdot r_{r}^{T_{r}} / T_{o r}^{12}}{3}
$$

With

$C_{\mathrm{Pr}}^{v}=D_{1}+D_{2} \cdot T_{r}+D_{3} \cdot T_{r}^{2}(26)$

\subsection{Mixture state:}

\subsubsection{Liquid state:}

$G_{r}^{l}=(1-X) \cdot G_{r H 20}^{l}+X \cdot G_{r N H 3}^{l}+T_{r}[(1-X) \cdot \ln (1-X)+X \cdot \ln X]$

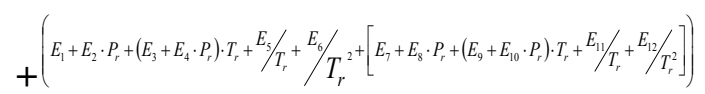

$+(2 X-1)+\left[E_{13}+E_{14} \cdot P_{r}+E_{15} / T_{r}+E_{16} / T_{r}^{2}\right](2 X-1)^{2} X(1-X)$

\section{3 .2 Gaseous state:}

\footnotetext{
Corresponding author: dardouch81@gmail.com
}

$G_{r}^{v}=(1-Y) \cdot G_{r H 20}^{V}+Y \cdot G_{r N H 3}^{V}+T_{r}[(1-Y) \cdot \ln (1-Y)+Y \cdot \ln Y](28)$

\section{Results and discussion}

We propose in this part, the results obtained from the simulations of the operation of the solar machine with and without distillation column.

The area of the solar collectors required to produce a cooling effect of $1 \mathrm{~kW}$ is shown in Figure 3 . The COPS in terms of Ts is illustrated in Figure 4.

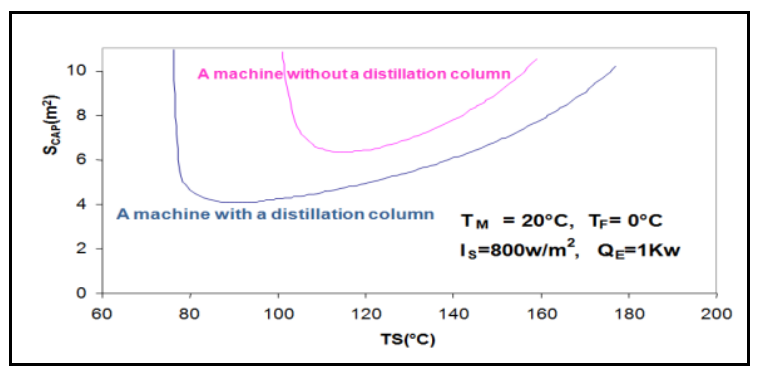

Fig. 3.Evolution of the surface of the solar collectors for the two types of machines with and without a distillation column in terms of Ts

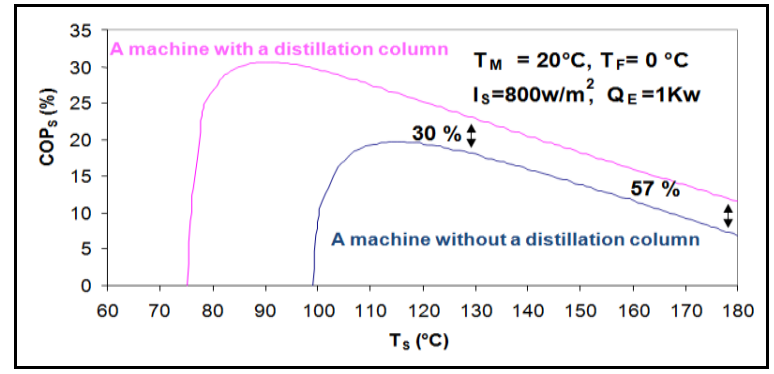

Fig.4.Evolution of the solar performance of coefficient for the two types of machines with and without a distillation column in terms of TS.

It can be seen from this curve that the use of a distillation column reduces the area of the solar collectors by $30 \%$.

It is noted that the curves shown in Figure 4have the same pace, except that the maximum is slightly shifted towards the low values of Ts.

\section{Conclusions}

The comparative study shows that the machine with distillation column is best suited to solar energy because it can be powered by simple flat plate collectors that are available and cheaper.

\section{References}

1. A. Bernatchou, M. Charia, J. Bougard,Possibilités de substitutions du R11, 
du R113 et R112 par le R133B1 et le R132B2, Rev. Gén. de Ther.,17-21 (1992).

2. O.M. Wageiallah,G. Yanling, Yearly Energetic and Exergetic Performance of Solar Absorption Refrigeration System In the Region of Northern Sudan, Int. Ene. Jou.,17, 141-154 (2017).

3. D. Wen Sun, Computer simulation and optimisation of ammonia-water absorption refrigeration systems, Ener. Sour.,19,677690 (1997).

4. V. Velmurugan, S.R. RajaBalayanan, K. SurendhraBabu, D. Sakthivadivel, Investigation of a Novel Solar Powered Absorption Refrigeration System with Solar Point Collector, Res. Jou. ofChe. Sci., 1, 5157 (2011).

5. D.S. Kim, C.A. Infante Ferreira,Solar refrigeration options - a state-of-the-art review, Int. Jou. of Ref., 31,3-15 (2008).

6. S. Bolocan, F. Chiriac, A. Serban, G. Dragomir, G. Nastase, Development of a small capacity solar cooling absorption plant, Ene. Pro. 74, 624-632 (2015).

7. Y. Boukhchana, A. Fellah, A. Ben Brahim, Modélisation de la phase génération d'un cycle de réfrigération par absorption solaire à fonctionnement intermittent, Int. Jou. of Ref., 34,159-167 (2011).

8. A. Allouhi, T. Kousksou, A. Jamil, Y. Agrouaz, T. Bouhal, R. Saidur, A. Benbassou, Performance comparison evaluation of solar air-conditioning systems in morocco, App. Ene. 170, 232-241 (2016).

9. S. Bahria, M. Amirat, A. Hamidat, M. El Ganaoui, M. Slimani, Parametric study of solar heating and cooling systems in different climates of Algeria - a comparison between conventional and high-energy-performance buildings, Ener. 113, 521-535 (2016).

10. A. Hamza , H. Ali, P. Noeres, C. Pollerberg,Performance assessment of an integrated free cooling and solar powered single-effect lithiumbromide-water absorption chiller, Sol. Ener. 82, 1021-1030 (2008).

11. C. Fathi, C. Guemimi, S. Ouaskit, An irreversible thermodynamic model for solar absorption refrigerator, Ren. Ene.,29, 1349. 1365 (2004).

12. R. Singh, R. Kumar, Theoritical Analysis of Nh3-H2o Refrigeration System Coupled With Diesel Engine: A Thermodynamic Study, 11, 29-36(2014).

13. S. Raghuvanshi, G. Maheshwari, Analysis of Ammonia-Water (NH3-H2O) Vapor Absorption Refrigeration System based on First Law of Thermodynamics, Int. Jou. of Sci. \& Eng. Res., 2, 01-05(2011).
14. A.Keçeciler, H.Acar, A.Dogan, Thermodynamic analysis of the absorption system with geothermal energy: an experimental study, Ene. Conv. and Man., 41,37-48 (2000).

15. M. Thioye,Amélioration de la performance des machines frigorifiques à absorption par l'utilisation de cycles à absorption et désorption étagés, Int. Jou. of Réf., 20, 136145 (1997).

16. J. Bougard,Applications de l'énergie solaire dans le froid et le conditionnement d'air, Int. Jou of Réf., 6, 319-328 (1983).

17. M.I. Karamangil, S. Coskun, O. Kaynakli, N. Yamankaradeniz, A simulation study of performance evaluation of single-stage absorption refrigeration system using conventional working fluids and alternatives, Ren. \&Sus. Ene. Rev., 14, 1969-1978 (2010).

18. J. Fernàndez-Seara, J. Sieres, the importance of the ammonia purification process in ammonia-water absorption systems, Ene. Conv. and Man., 47, 1975-1987 (2006).

19. RM. Lazzarin, A. Gasparella, GA. Longo, Ammonia-water absorption machines for refrigeration: theoretical and real, Int. Jou. ofRéf., 19 ,239-246 (1996).

20. N. Chekir, Kh. Mejbri, A. Bellagi, Simulation d'une machine frigorifique à absorption fonctionnant avec des mélanges d'alcanes, Int. Jou. of Réf., 29, 469- 475 (2006).

21. V.Srikanth, B. Raja Narender, Dr.A. Guptan, thermodynamic analysis of vapour absorption refrigeration system using solar energy, Int.Jou.ofLat. Tre.in Eng.\& tech., 7, 17-26 (2016).

22. J. Dardouch, M. Charia, A. Bernatchou, Study of an Absorption Refrigeration Machine Improved with Distillation Column, Jou. of Mat. andEnv. Sci., 9, 772-783 (2018).

23. M. Barhoumi, A. Snoussi, A. Ben Ezzine, K. Mejbri, A. Bellagi, Modelling of the thermodynamic properties of the ammonia water mixture,Int. jour. of Ref., 27 , 271-283 (2004).

24. M. Ahachad, A. Almers, A. Mimet, A. Draoui, Solar-based comparison of adsorption and absorption refrigerating machine, Int. Jour. of Sust. Ene., 24, 199-206 (2005).

25. M. Benramdane, Y. Khadraoui, S. Abboudi, Amélioration des performances des installations de réfrigération solaires à absorption, Rev. des Ene. Ren. SIENR, 12, 357-365 (2012).

26. M. Charia, A. Pilatte, M. Bouidida, Machine frigorifique à absorption (eau - ammoniac) fonctionnant avec des capteurs plans sur le site de Rabat, Rev. Int. de Froi., 14, 297-303 (1991). 
27. M. Ahachad, M. Charia, A. Bernatchou, Solar absorption heat transformer Applications to absorption refrigerating machine, Int. Jour. of Ene. Res., 17, 719-726 (1993).

28. B. Chaouachi, S. Gabsi, Design and Simulation of Absorption Solar Refrigeration Unit, Ame. Jour. of App. Sci., 4, 85-88 (2007).

29. A. Machrouhi, M. Charia, A. Bernatchou, Perforormance of a vapour compression system functioning with the hydrocarbons 1,4 pentadiene and cyclobutane, Int. Ref. and Air Cond. Conf., 461-465 (1998).

30. R. Fathi, S. Ouaskit, Performance of a Solar LiBr-Water Absorption Réfrigération Systems, Rev. des Ene. Ren.: journées de thermique, 73-78 (2001).

31. M. Hamatti, R. Tadili, M.N. Bargach., A. Mechaqrane, B. Ihya, Evaluation des composantes spectrales du rayonnement solaire à Rabat (Maroc), Rev. Int. d'hél. 44, 1-6 (2012).

32. H. Essalhi, R. Tadili, M.N. Bargach, Conception et Expérimentation d'un Capteur Solaire à Air pour un Séchoir Solaire Indirect, Int. Jour. of Sci. Res. \&Eng Tech. 4, 78-81 (2015).

33. S. Kherris, M. Makhlouf, D. Zebbar, O. Sebbane,contribution study of the thermodynamics properties of the ammoniawater mixtures, Rev. Ther. Sci, 17, 891-902 (2013).

34. M. Benramdane, A. Ghernaouet, S. Abboudi,Contribution to Improving the Performance Coefficient of a Solar Absorption Refrigeration System, Int. Jou. Of Res. \& Rev .Appli. Sci., 21,71-81 (2014).

35. M. Barhoumi, A. Snoussi, N. Ben Ezzine, Kh. Mejbri, A. Bellagi, Modelling of the thermodynamic properties of the ammonia/water mixture, Int. Jou. ofRéf., 27, 271-283 (2004).

36. B. Ziegler, Ch. Trepp, Equation of state for ammonia-water, Int. Jou. Of Réf.,7, 101-104 (1984). 